\title{
Removal of internal multiples with the common-focus-point (CFP) approach: Part 2 - Application strategies and data examples
}

\author{
D. J. Verschuur ${ }^{1}$ and A. J. Berkhout ${ }^{2}$
}

\begin{abstract}
In the past, the surface-multiple-removal method based on the feedback model has been successfully applied to many different field data sets. The extension of surface to internal multiples can be made by replacing shot records with common-focus-point (CFP) gathers, a CFP gather representing focused data with one source in the subsurface and all receivers at the surface (or vice versa for a receiver gather). The internalmultiple-removal algorithm can be formulated in terms of boundary-related and layer-related versions. In the boundary-related version, the internal multiples are removed for one downward-scattering reflector at a time. In the layer-related version, the internal multiples are removed for a sequence of downward-scattering reflectors at a time. An exact velocity model is not required, but proper muting is critical; muting becomes straightforward in the CFP domain. The strategy for applying the two versions of the multiple-removal algorithm is demonstrated on physical-model and field data. One can conclude that the layer-related version is the most appropriate in most situations because it requires less user action and does not need exact knowledge of the multiple-generating boundary.
\end{abstract}

\section{INTRODUCTION}

During the last decade, the interest in multiple-removal techniques has grown significantly. One of the reasons is that the problem of multiples in seismic (marine) data has not yet been solved and can be a major hurdle in 3D processing flows. The major advantage of using wave-theory-based multipleprediction methods is that statistical filters are only involved in the subtraction stage and not in the prediction stage (as, e.g., Wiener prediction-error filtering). In Berkhout (1982), the extension from surface to internal multiples is described by including downward extrapolation, and in Berkhout and Verschuur (1997) this idea is reformulated by replacing shot records with common-focus-point (CFP) gathers. In Berkhout (1999), this concept is generalized by also considering the internal multiples generated by a complete layer instead of a single interface. Note that a CFP gather is obtained by focusing surface data on the source (or receiver) side, resulting in a new gather with one source in the subsurface and all receivers at the surface (or vice versa) (see Berkhout, 1997a; Thorbecke, 1997).

A field-data example on boundary-related internal-multiple removal is shown by Hadidi and Verschuur (1997). Kelamis et al. (2002) demonstrate boundary-related and layer-relatedmultiple removal in the poststack and CMP domains. From the inverse-scattering point of view, a field data example of internal-multiple removal is presented by Matson et al. (1999). In the inverse-scattering approach (Weglein et al., 1997) all possible multiple-generating boundaries are treated in a stepwise, automatic procedure.

In this paper, a strategy for applying the boundary-related and layer-related approach to internal-multiple removal is given. Practical aspects are discussed, and the algorithms are demonstrated on physical model data and field data.

\section{REMOVAL OF INTERNAL MULTIPLES}

The theory for surface-related multiples (Riley and Claerbout, 1976; Kennett, 1979; Berkhout, 1982; Verschuur, 1991; Berkhout and Verschuur, 1997) can be extended for internal multiples (Berkhout, 1982, 1999). The data-driven spatial convolutions, however, do not take place at the surface but at the multiple-generating interface. Therefore, inverse propagation operators are required to extrapolate the seismic data toward the internal-multiple-generating boundary. These operators are called focusing operators in the remainder of this paper

\footnotetext{
Manuscript received by the Editor November 6, 2002; revised manuscript received November 14, 2003; published online May 23 , 2005.

${ }^{1}$ Delft University of Technology, Faculty of Applied Sciences, P.O. Box 5046, 2600 GA Delft, The Netherlands. E-mail: d.j.verschuur@ tnw.tudelft.nl.

${ }^{2}$ Delft University of Technology, Faculty of Technology, Policy and Management, P.O. Box 5015, 2600 GA Delft, The Netherlands. E-mail: a.j.berkhout@tbm.tudelft.nl.

(c) 2005 Society of Exploration Geophysicists. All rights reserved.
} 
and can be considered as time-reversed Green's functions that describe the propagation between the surface and one focus point in the subsurface. These focusing operators can be determined in a fully data-driven manner without explicitly using subsurface information, transforming shot records into CFP gathers (see Morton, 1996; Berkhout, 1997b; Thorbecke, 1997; Bolte and Verschuur, 1998). Thus, propagation between the surface and the interface under consideration is directly described in terms of wavefield operators, and not in terms of velocity-depth parameters.

In Figure 1a, the construction of surface multiples is demonstrated for one source-receiver pair by combining traces from one common-shot record with traces from one commonreceiver gather in a surface-consistent way. The same process can be organized per surface reflection point (right-hand side, Figure 1a). Using a common-source gather and a commonreceiver gather for the same surface point, the multiple contribution from this surface location to all source-receiver pairs can be constructed. For surface-related multiple prediction, this organization does not have a clear advantage.

In Figure 1b, a similar construction is observed for internal multiples, but now the traces are selected from CFP gathers, where either sources or receivers are located at the multiplegenerating boundary. Organizing the process per subsurface reflection point (right-hand side, Figure 1b) shows that the prediction algorithm can be applied in a practical manner, CFP gather by CFP gather. For each subsurface location, using one CFP gather, the contribution to the predicted multiples for all surface locations is computed. This is the implementation used for the examples: first, construct CFP gathers; then calculate from each CFP gather the internal-multiple contributions to all desired seismic traces.

Berkhout (1999) proposes to replace the boundary-related approach with a layer-related approach. In this way, the downward-reflecting effects of a complete overburden can be taken into account. A complete description of this approach can be found in Berkhout and Verschuur (2005), hereafter referred to as part 1 . In terms of implementation, this means that besides CFP gathers, a redatumed version of the CFP gathers - with source and receivers at the chosen depth level - is also needed. From these so-called gridpoint gathers, the causal part of the time-reversed version is used to predict in a datadriven manner the downward-reflecting effects of the entire layer (see Figure 1c).

For both internal-multiple-removal approaches, muting of the data prior to multiple prediction plays an important role: all events reflecting at boundaries above and including the multiple-generating reflector under consideration must be removed from the data before prediction is applied. This can be compared to removing direct and surface waves from the shot records in the case of surface-related multiple removal. In the CFP domain, this muting is a straightforward process because the first arrivals of the used focusing operator can be used directly as a mute line. This is described in part 1 .

Furthermore, part 1 shows that the boundary-related approach requires focusing operators to be exact. If a velocity model of the subsurface is not available, the focusing operators must be determined with an operator-updating procedure, as described by Berkhout (1997b) and Thorbecke (1997). In this updating procedure, the operators are updated based on the principle of equal traveltime: the correct focusing operator has exactly the same traveltimes as the corresponding reflection event in the CFP gather. By constructing differential time shift (DTS) panels, we find that the event related to the reflection point of interest should be located at zero differential time for all CFP offsets. A DTS panel is created by taking the CFP gather and subtracting from each trace the corresponding focusing-operator traveltime. If the focusing operator was not correct, this DTS panel will show an event that is not a zero differential time for all surface locations. Any residual traveltime error can be fed back into the operator, after which a new CFP gather can be calculated. This procedure is repeated iteratively for each CFP location at the boundary until all DTS panels show a flat event at zero differential time, 
indicating correct operators. Starting with initial operators based on the NMO velocities, this process typically requires two to three iterations.

However, the layer-related approach relaxes this constraint considerably: only an approximate velocity model need be defined because velocity errors will cancel during the multipleprediction process. Thus, user interaction is restricted to picking an approximate time level in a stack or time migration, and stacking velocities can be used to construct CFP gathers and gridpoint gathers.

Finally, note that the boundary-related internal-multipleprediction process involves only one downward continuation (i.e., focusing) and one lateral convolution step, whereas the layer-related process involves two focusing steps and two lateral convolutions, which makes it twice as costly in terms of computation time.

\section{BOUNDARY-RELATED AND LAYER-RELATED REMOVAL STRATEGY FOR MULTIPLES}

The choice of whether to use the boundary-related or the layer-related strategy depends on the type of internal multiples to be removed. For this purpose, several internalmultiple-generating situations are analyzed.

As a first situation, Figure $2 \mathrm{a}$ is considered. It resembles a strong reflector on top of a moderately reflecting medium. In this case, the first reflector (the water bottom) is a strong downward scatterer, with reflection strength $-R$; all reflectors below the water bottom are assumed to have weaker strength $r$. All internal multiples generated entirely below the first reflector have a reflected amplitude of order $r^{3}$, and all internal multiples related to the first reflector have a strength of order $R r^{2}$. Note that, with the same reasoning, surface-related multiples are typically one order stronger in amplitude compared with internal multiples. For the situation of a simple strong water-bottom reflection, the choice for the boundary-related approach - the water bottom being the multiple-generating interface - is straightforward: the propagation operators between the water bottom and the surface are easily constructed from available water depth and velocity information or from picking the event in a (time) migrated section.

Next, consider the situation of Figure 2b: one strong reflector is embedded within reflectors with smaller reflectivity. This reflector generates three types of first-order internal multiples. The first type of multiples are relatively strong ones, as shown in the left of Figure 2b, with two upward bounces at the strong reflector and one downward bounce at one of the reflectors above, the reflection strength being $r R^{2}$. The second and third types have only one upward or downward bounce at the strong reflector, the reflection strength being $R r^{2}$ (the second and third raypaths in Figure 2b). Multiples of the first, strongest type can be removed with the boundary-related approach by scanning all boundaries above the strong reflector. In Figure 2b, this means four boundaries. This also removes internal multiples of the third type. Then, the strong boundary itself needs to be processed as well in a next boundary-related step to remove all multiples that are $R r^{2}$. As indicated with the two levels $A$ and $B$ in Figure 2b, only two layer-related steps are needed to predict all of these internal multiples. Furthermore, applying the two steps eliminates the need to define and update focusing operators for many boundaries. In such a situation, the layer-related approach is always preferred.

In practice, the internal-multiple problem is always a mixture of the above-described situations, as shown in Figure 2c, where two strong reflectors are embedded in softer sediments. This situation can, for example, occur in a salt or basalt layer. If only the multiples generated between these two strong reflectors are the objective of multiple removal (i.e., the middle type displayed in Figure 2c), the boundary-related procedure may still be a viable option. If the focusing operators can be updated with little effort, the boundary-related approach requires less data manipulation than the layer-related approach.

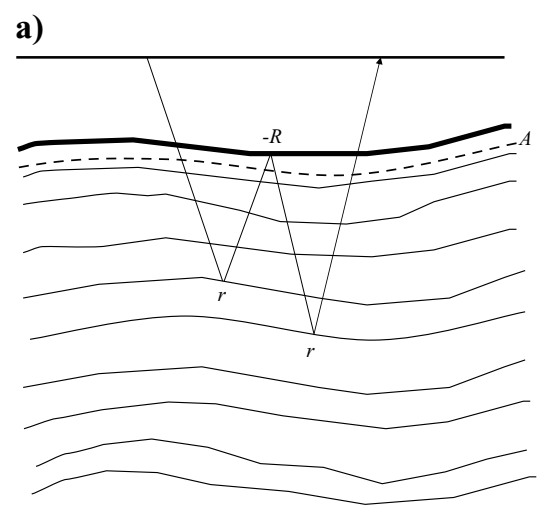

b)

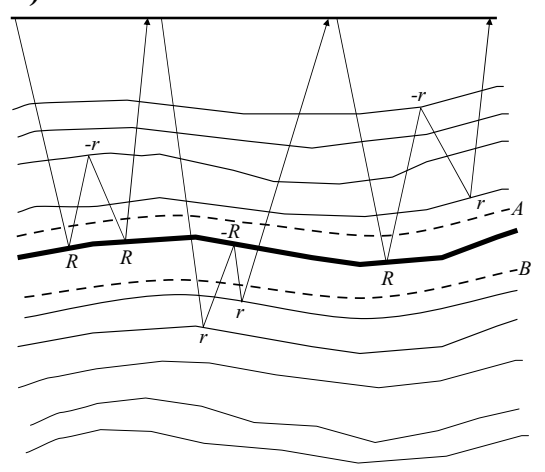

c)

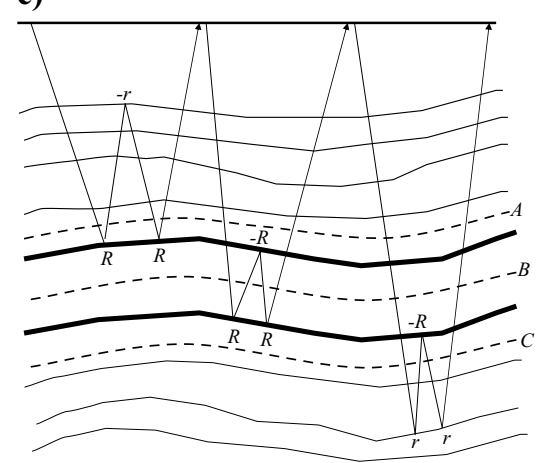

Figure 2. Three situations of internal-multiple generation. (a) Internal multiples related to the water bottom. (b) Internal multiples from a strong reflector embedded in softer sediments. The strongest multiples are generated by a downward bounce above the strong reflector. (c) A strong reflective layer embedded within softer sediments. The leftmost multiple type is stronger than the rightmost multiple type. 
This is demonstrated in the first field-data example in this paper. However, if all internal multiples that are in some way generated at these strongly reflecting boundaries need to be removed, a number of strategically defined layers (always just above or below the strong boundaries) is better. The last fielddata example in this paper will demonstrate this.

Note that the layer-related internal-multiple-removal method will not correctly handle internal multiples that are generated completely within this layer. Therefore, one layer in the

a)

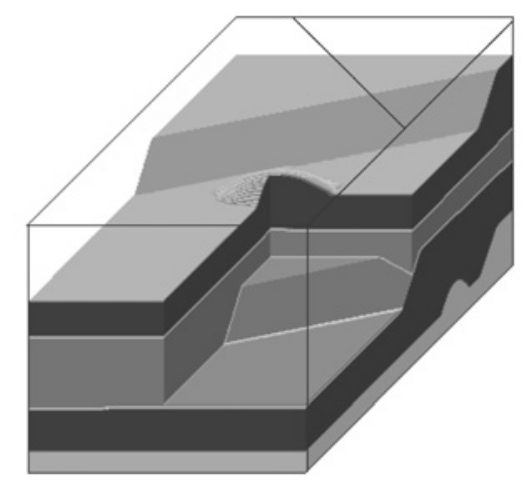

b)

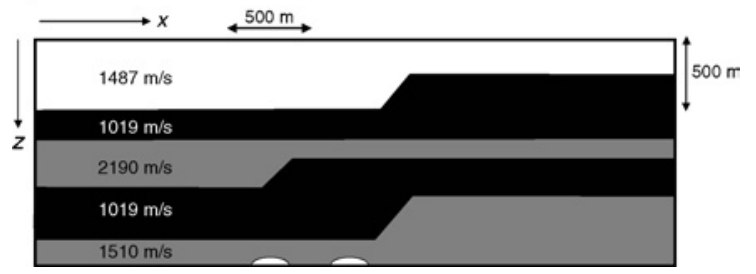

Figure 3. (a) A 3D subsurface model used to acquire seismic physical model data in the Delft Experimental Facility for Imaging. The line drawn along the surface indicates the approximate position of the $2 \mathrm{D}$ seismic line. It is chosen to minimize out-of-plane effects. (b) A schematic cross section of the model along the seismic profile.

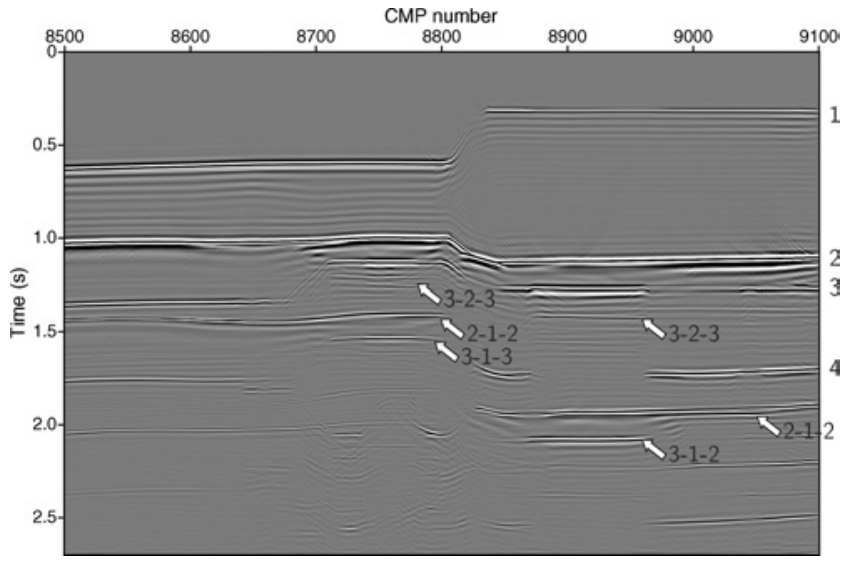

Figure 4. Time-migrated stack of a data set measured in the Delft physical modeling facility. The arrows indicate internal multiples related to the first and second boundaries. The sequence of bounces is indicated. layer-related approach can never contain two strong boundaries.

Finally, even if the important internal multiples are generated by downward reflection at one specific boundary but the boundary has a complex structure (e.g., a complex waterbottom or salt boundary), then the layer-related route is always advised, with the level chosen below the boundary (see level $A$ in Figure 2a). The latter is demonstrated in part 1, where the water-bottom reflector is represented by two closely separated reflectors. Moreover, as already mentioned, the layer-related approach is robust to kinematic errors in the focusing operators.

\section{PHYSICAL MODEL TANK DATA EXAMPLE}

For the first example, a data set measured in the Delft Experimental Facility for Imaging (EFI) is considered. For a more elaborate description of this physical modeling tank, see Koek et al. (1995) and Blacquière et al. (1999). The data under consideration are acquired with a $3 \mathrm{D}$ geometry for the model shown in Figure 3a. The first, third, and fourth boundaries contain a fault, whereas the second boundary is completely horizontal. Furthermore, in interfaces 1 and 3, a dome structure is integrated, although the dome in interface 3 is not visible in Figure 3a. Figure $3 \mathrm{~b}$ is a schematic of a $2 \mathrm{D}$ cross section of the model along the sail line. As can be observed, below interface 4 some reservoir-like anticlinal structures are present. One streamer from one sail line is selected, and these data are considered as a 2D experiment. The streamer consists of 300 shots, each with 100 receivers. The source and receiver spacing is $25 \mathrm{~m}$. Because the two domes in the model within interfaces 1 and 3 are located far enough from the considered line, and the direction of the sail line is chosen perpendicular to the fault planes, the medium can be assumed to be locally two dimensional.

In Figure 4, the time-migrated stack of this line is displayed. Note the strong internal multiples generated within the thick first subbottom layer and between the second and third reflectors, indicated by the arrows. Additional internal multiples that are generated in the thin layer between the second and third reflectors are visible. In this model, gas cavities exist at the third interface (between CMPs 8700 and 8800 and CMPs 8870 and 8970 , where the layers are locally disconnected). This results in a locally strong reflection, making interbed multiples easily visible.

With this data set we show that both the boundary-related and layer-related methods can give similar results when applied in a consistent manner.

\section{Boundary-related approach}

Since this model has well-defined and well-separated interfaces, the data appear to be a good candidate for boundaryrelated internal-multiple removal. The multiples can be removed boundary by boundary (boundary stripping), starting with the shallowest multiple generator.

The water bottom is considered first. Because the water velocity was not exactly known, the time-migrated stack was picked and the assigned hyperbolic operators were updated to accurately describe the one-way water-layer propagation effects. Figure 5 illustrates the result of operator updating: the 
DTS panels after two updates show flat events at zero differential time.

All DTS panels related to a dense grid of CFP locations along the boundary of interest form a so-called DTS volume. The (nonaligned) event around zero time is represented in the DTS volume by a surface. This is what we use in practice to update the focusing operators.

In the first update, the traveltime errors are only roughly picked to compensate for the major part of the operator errors. This is done by picking a number of points in a few selected DTS panels and interpolating a smooth surface across these points. Figure 6a shows this smooth surface for the DTS panels from boundary 1 , based on the initial operators. Note that velocity errors are present, which can be observed as nonzero traveltime error values. At the steeply dipping flank (around CFP 350) the focusing operators show large errors. After one update, the operators are much closer to the correct ones, and a more detailed traveltime surface determination is required for the final update.

With the use of a multidimensional event tracker, detailed traveltime-error information is extracted from this DTS volume. The event tracker, based on the work of Spagnolini and Rampa (1999) and illustrated in Bolte et al. (2000), needs a few starting points only. The tracking algorithm is based on
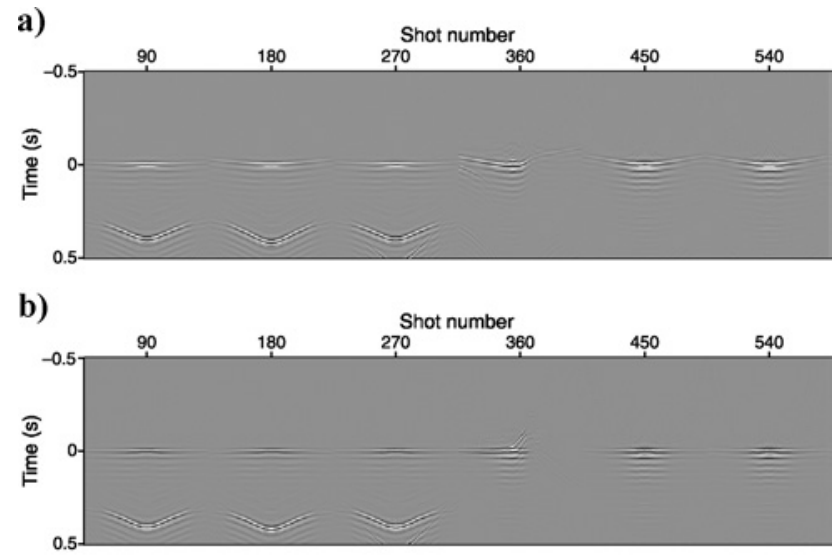

c)

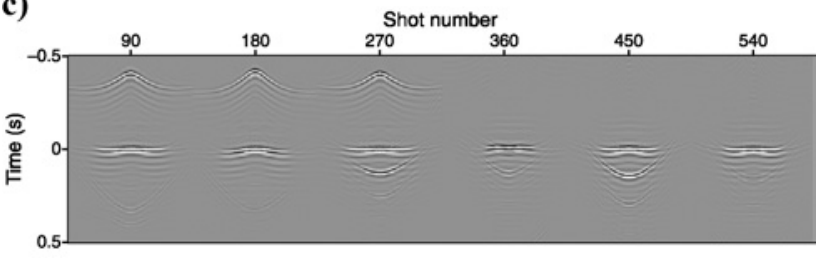

d)

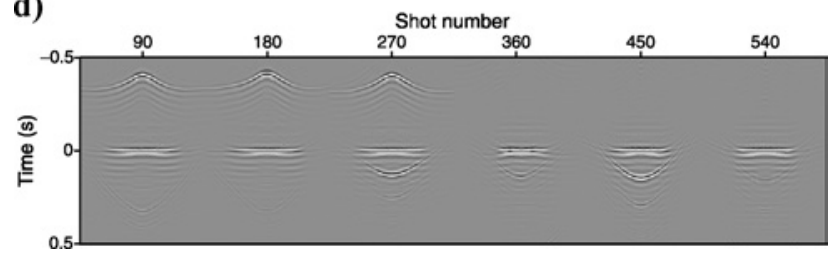

Figure 5. Result of focusing-operator updating for $(a, b)$ boundary 1 and $(\mathrm{c}, \mathrm{d})$ boundary 2 , showing initial $(\mathrm{a}, \mathrm{c})$ and final $(b, d)$ focusing operators. The flat events at zero time in the DTS panels after two updates in (b) and (d) indicate that the principle of equal traveltime is fulfilled. correlation between adjacent traces, and the tracked surface will always expand along points with the best correlation measure. The starting points are picked manually in some selected DTS panels, after which the tracker finds the complete error surface. Tracking continues only if the correlation between traces is above a certain threshold. Thus, bad data areas may not be tracked. The picked traveltime-error surface is shown in Figure $6 \mathrm{~b}$. Note again that the location of the fault in the original section is visible around CFP 350. By interpolation and extrapolation, operator-error times can be obtained for all focus-point locations and all CFP offsets. The actual updating in both iterations is carried out by dividing the traveltime error in all DTS panels by two and adding this traveltime error to the current operators. Note that in this process, no velocitydepth model is involved. This second update appears sufficient to flatten all panels (Figure 5b).

Next, the first event is muted from the CFP gathers from the last update cycle. After a lateral convolution of these muted CFP gathers, the internal multiples are predicted (bear in mind the right-hand side of Figure 1b). In Figure 7a-c, the CFP
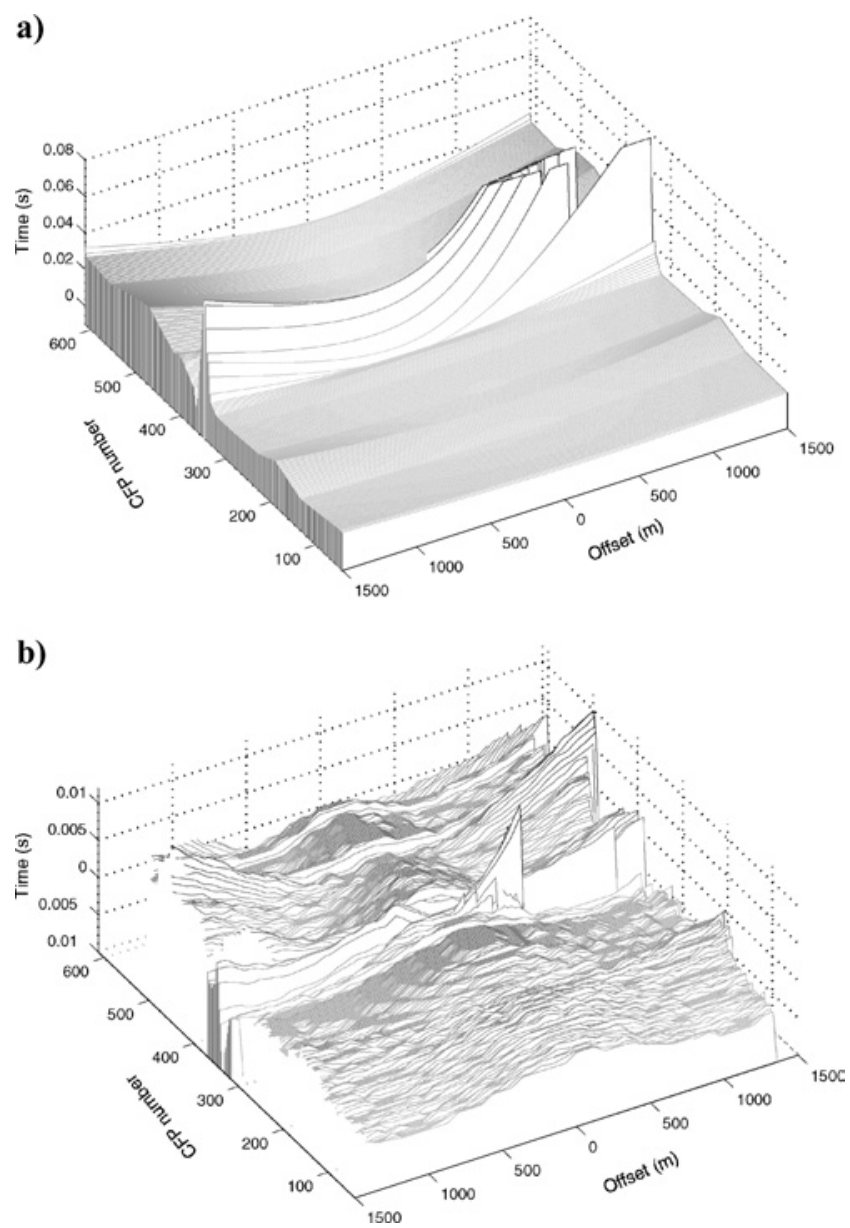

Figure 6. Using a multidimensional tracking algorithm, the traveltime errors can be found along the first boundary for each set of DTS panels, as shown in Figures 5a and 5b. The tracked traveltime-error surfaces are displayed in $3 \mathrm{D}$ view for (a) initial and (b) updated DTS volumes. These traveltime errors are used to update the focusing operators. Nontrackable parts of the DTS volume are interpolated. 
gather and one-shot gather with the resulting predicted multiples are displayed for one lateral location. By using CFP gathers for internal-multiple prediction, the resulting multiples are found at the surface locations. Thus, they can be subtracted from the original shot records. The CFP gathers are only used in the prediction stage and can be deleted afterward. After adaptive subtraction from the input shot, a good multiple suppression is obtained (Figure 7d).

The resulting shot records are stacked and time migrated (Figure 9a). The internal multiples related to the first reflector are well suppressed compared with the middle part of the input section of Figure 4.

A next boundary-related procedure involving the second reflector in the model handles the other visible internal multiples in the data. The second boundary is picked in the time section, and initial focusing operators are computed from these apex times by assigning hyperbolic one-way traveltime curves using the NMO velocities. The resulting DTS panels (Figure 5c) show that the operators need to be updated. After two iterations, flat events at zero time in the DTS panels are obtained (Figure 5d) and the updated focusing operators can be used to calculate the CFP gathers needed for the multiple prediction process.

a)

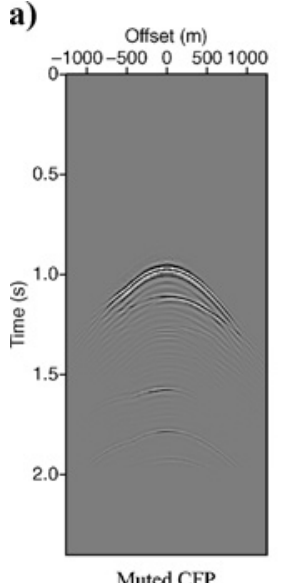

c)

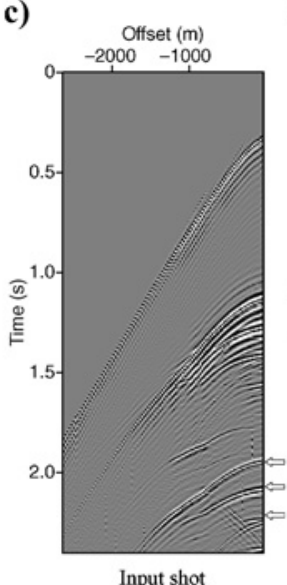

e)

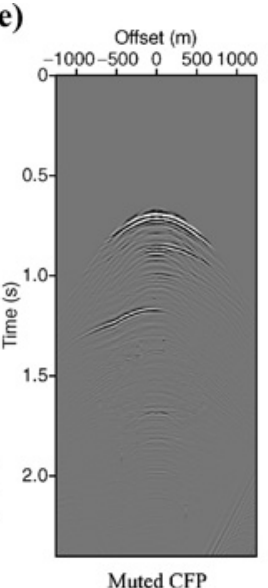

g)

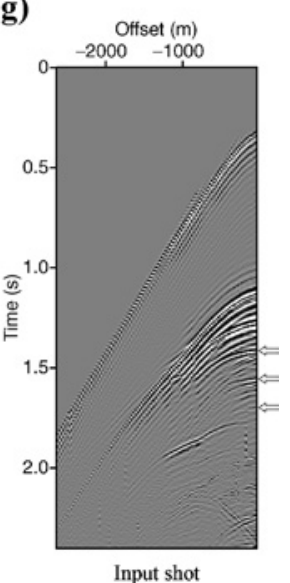

b)

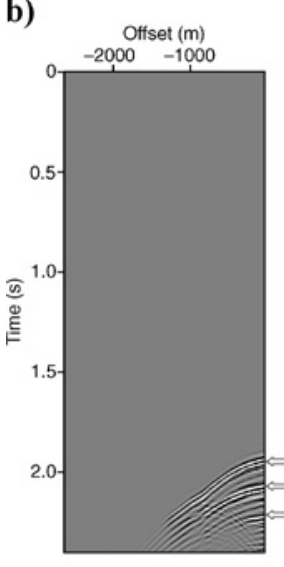

d)

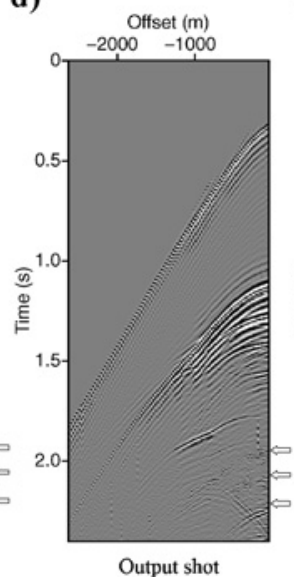

f)

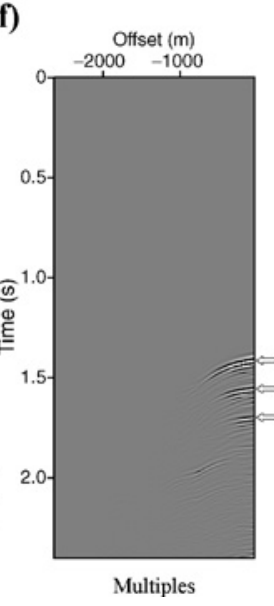

h)

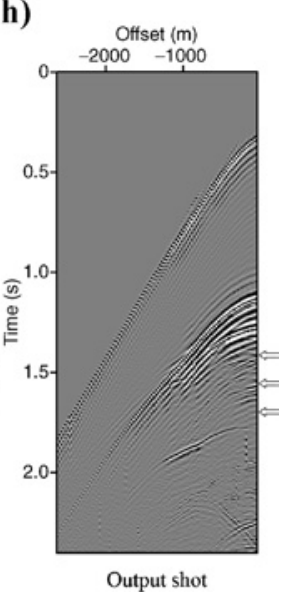
served (Figure 9b).

The CFP gathers from the last iteration are muted along the operator times to remove the events from the first two boundaries. Next, they are used in the internal-multiple prediction process. Figures $7 \mathrm{~d}-\mathrm{g}$ show the results for one muted CFP gather and one shot gather. As expected, multiples related to the second boundary are suppressed as well. At stack level, the reduction of internal multiples can be readily ob-

\section{Layer-related approach}

As demonstrated, the focusing operator updating process is facilitated by automatic multidimensional trackers to obtain the traveltime error function for each DTS panel. However, user interaction remains necessary. To obtain a faster turnaround time, the results of the two boundary-related procedures can be achieved equally well by two more robust layer-related procedures: the lower boundary of the first layer is positioned between the first and second reflections, and the lower boundary of the second layer is positioned between the second and third reflections.

First, focusing operators are determined using the vertical traveltimes of the chosen time level and using NMO velocities. Next, CFP gathers are created for a regularly sampled set of locations along this time level. The resulting prestack volume of CFP gathers is muted by using the operator times. This removes all reflections from above the defined level. In Figure 8a, a muted CFP gather is shown for one CFP location.

Then, the CFP gathers from the previous step - but without the mute - are used as input for a second focusing step with the same focusing operators. The resulting gridpoint gathers describe fully redatumed shot records with sources and receivers at the defined time level. The events in these gathers at positive times are the reflections from below this level; at negative times, the reflections from above this level are present but are seen from below. By time-reversing these gathers and selecting the causal part, the upward transfer functions are obtained. For the same selected position, the time-reversed and muted gridpoint gather is displayed in Figure $8 \mathrm{~b}$. The multiple-generating reflection above the level is visible. As can be observed in Figure 1c, internal multiples related to the involved level are now constructed by a spatial and temporal convolution of the muted CFP gathers with the time-reversed gridpoint gathers, followed by another spatial and temporal convolution with a reciprocal version of the muted CFP gathers. Note that in these procedures, no operator updating is involved. The use of NMO velocities is sufficient because the errors in the creation of CFP gathers are compensated by the opposite

Figure 7. One muted CFP gather and one shot record before and after boundaryrelated internal-multiple removal for boundary $1[(\mathrm{a})-(\mathrm{d})]$ and boundary $2[(\mathrm{e})-(\mathrm{h})]$. The input shot for the second step (g) is the output shot of the first step (d). The arrows point to the location of some internal multiples. 
errors in the gridpoint gathers. The predicted internal multiples have sources and receivers at the surface and can be directly matched with the original shot records. The predicted multiples for one shot position are displayed in Figure 8c. Adaptive subtraction from the input shot yields a result very similar to the boundary-related version (compare Figure 8e with Figure 7d).

The wavelet of the predicted multiples will be different for the boundary- and layer-related versions. In the boundaryrelated version, as a result of the autoconvolution of the seismic data, the predicted multiples will contain the autoconvolution of the wavelet (similar to surface-related multiple prediction). In the layer-related version, there is one convolution with a CFP gather and one with the time-reversed gridpoint gather. For the resulting wavelet in the predicted multiples, this means the phase of the wavelet is the same as the input data, but the amplitude spectrum is the original amplitude spectrum to the third power. The latter is also observed in the internal-multiple-removal approach described by Jakubowicz (1998), which involves a convolution and a correlation step. An adaptive subtraction of the predicted multiples from the input data correct for these wavelet effects.

The resulting multiple-suppressed shot records are then used in a second layer-related process, now considering multiples generated between the second and all deeper boundaries. Figure $8 \mathrm{f}$ and $\mathrm{g}$ shows one CFP gather and one gridpoint gather involved in the multiple-prediction process, and Figure $8 \mathrm{~h}$ and $\mathrm{j}$ shows the result of multiple subtraction for one shot record. Note from the time-reversed gridpoint gather (Figure $8 \mathrm{~g}$ ) that, besides the reflections at negative times, the first reflection needs also to be muted because multiples related to this boundary have already been removed. Note also that the two layer-related steps cannot be replaced by one layer-related step at level 2 , only because internal multiples generated within this layer (i.e., multiples between reflectors 1 and 2) are otherwise not addressed - only multiples between reflectors 1 or 2 and reflectors 3, 4, and 5, etc.

In the poststack migrated domain, one can observe that the results of layerrelated internal-multiple removal (Figure $9 \mathrm{c}$ and $\mathrm{d}$ ) are very similar (and even slightly better) than their boundaryrelated counterparts (Figure 9a and b). However, the layer-related approach is considerably simpler.

\section{FIELD-DATA EXAMPLE OF BOUNDARY-RELATED APPROACH}

For a demonstration of boundaryrelated internal-multiple removal on field data, a North Sea field data set from the Haltenbanken Terrace (courtesy Saga

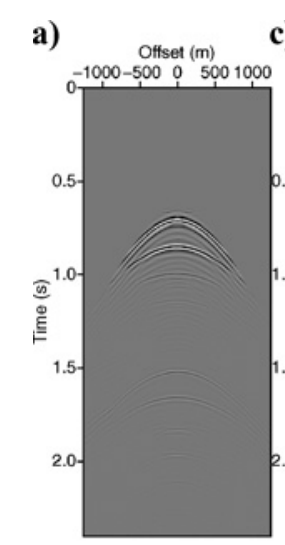

a)

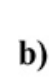

b)

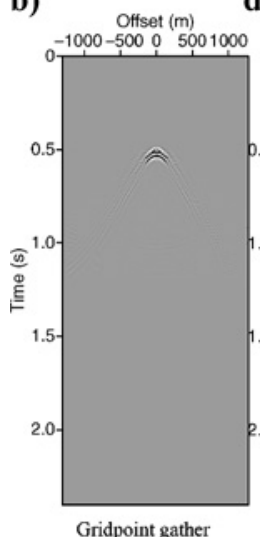

Gridpoint gather
Petroleum A.S.) is considered. After surface-related multiple removal, an internal-multiple contamination is observed based on comparing the surface-multiple removal output with well-log information. This is the same data set from which Verschuur et al. (1992) and Verschuur and Berkhout (1997) successfully remove the surface multiples.

As a first step, the multiple-generating interface is indicated in a time section. For the North Sea field data set, this is shown in Figure 10 by the traveltime track displayed on the stack around $2.3 \mathrm{~s}$ (at the arrows). We assume this subbottom reflector to be the first relevant multiple generator. Since the velocity-depth model is unknown at this processing stage, generating the correct focusing operators requires an iterative updating procedure. Based on NMO velocity information, initial one-way traveltime operators are created to be verified (Figure 11a). Remember that each DTS panel reveals the validity of its one-way propagation operator from one subsurface location (i.e., one dot in the traveltime track in Figure 10) toward all surface locations. Based on the initial operators, the event at zero differential time is not flat, and updating of the focusing operators is required. As shown in the physical-model data example, the traveltime error can be obtained from the DTS panels by using an automatic tracker. The obtained traveltime-error surface in the first iteration c)

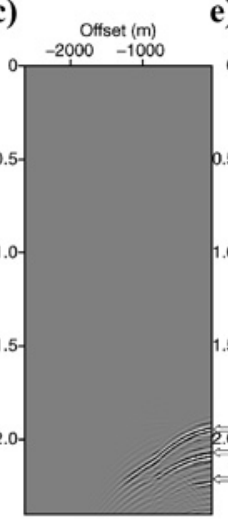

Multiples

d)

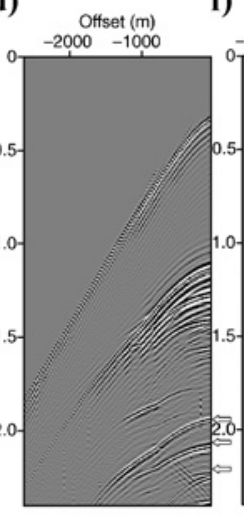

Input shot e)

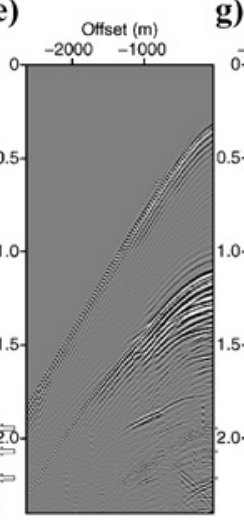

Output shot

f)

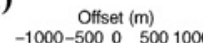

g)
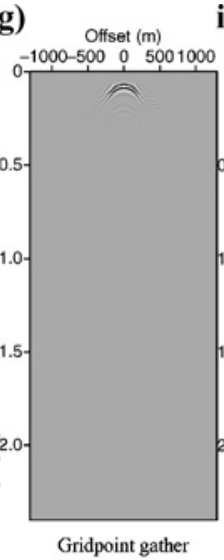

h)

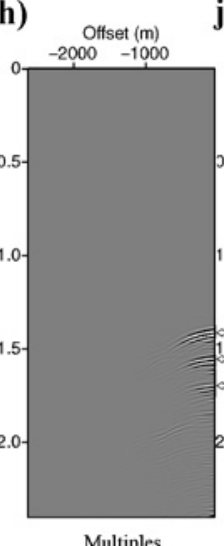

Multiples i)

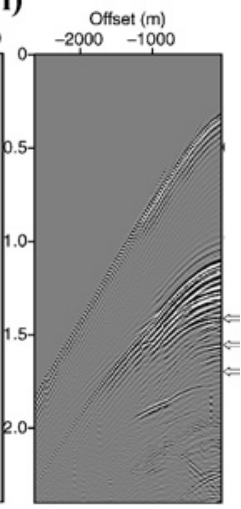

Input shot j) Offset (m)

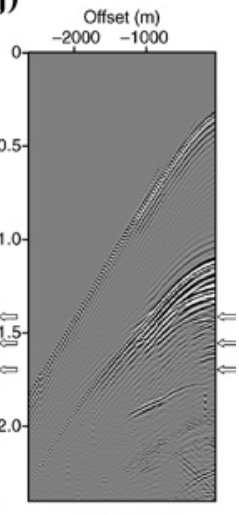

Output shot

Figure 8. One muted CFP gather, one time-reversed and muted gridpoint gather, and the results before and after layer-related internal-multiple removal for level 1 [(a)(e)] and level 2 [(f)-(j)]. The input shot for the second step (i) is the output from the first step (e). The arrows indicate the location of some internal multiples. 
a)

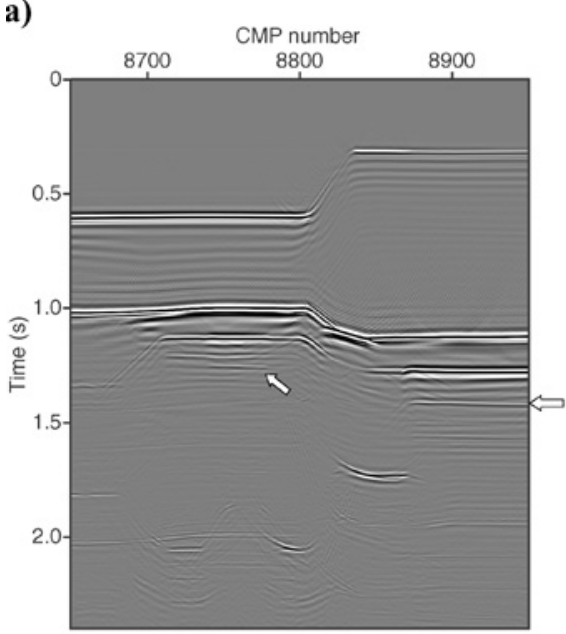

b)

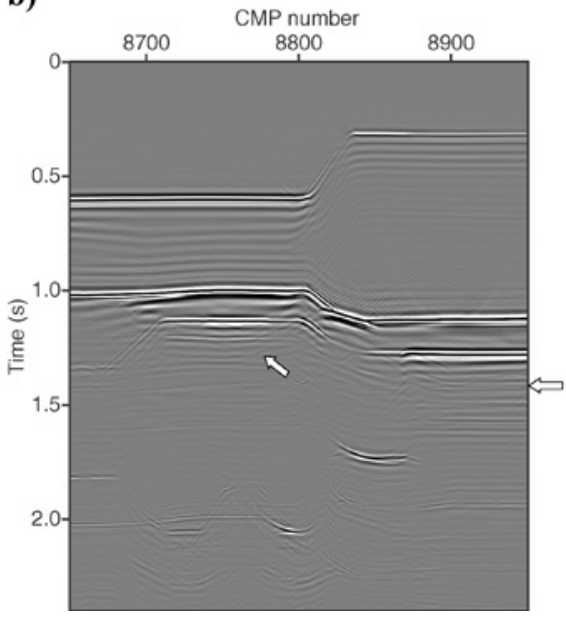

c)

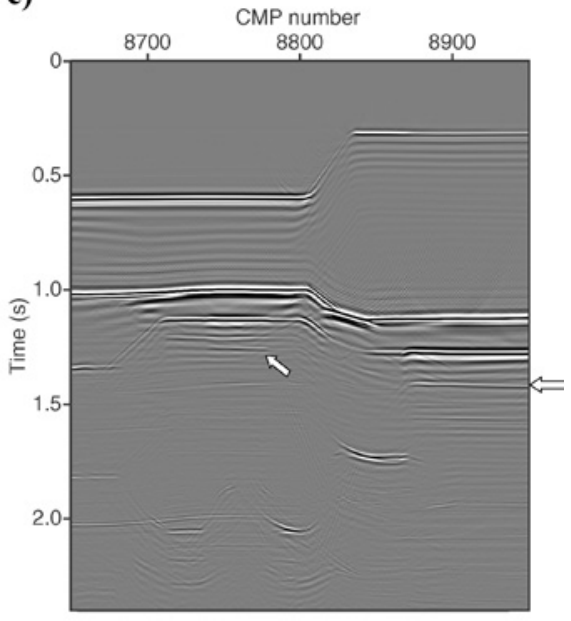

d)

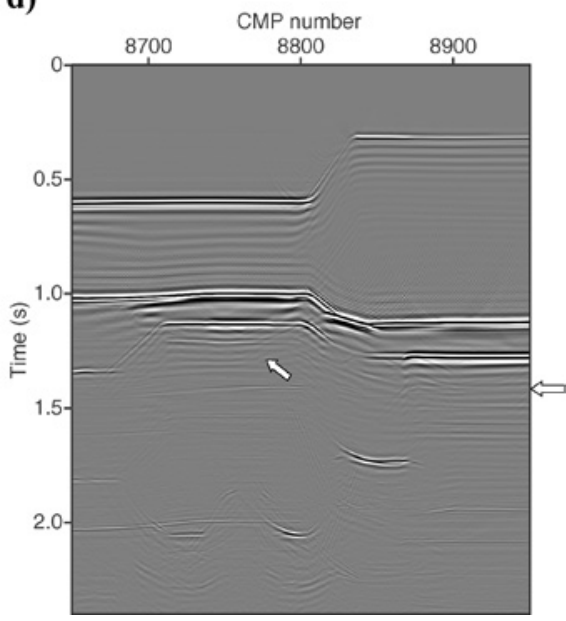

Figure 9. Time-migrated stack of the physical-model data after four internal-multiple removal steps. The middle part of the stack in Figure 4 represents the input data with multiples. The arrows indicate internal multiples related to the second reflector. Note the large similarity between the boundary-related [(a) boundary 1; (b) boundary 2] and the corresponding layer-related [(c) boundary 1; (d) boundary 2] results.

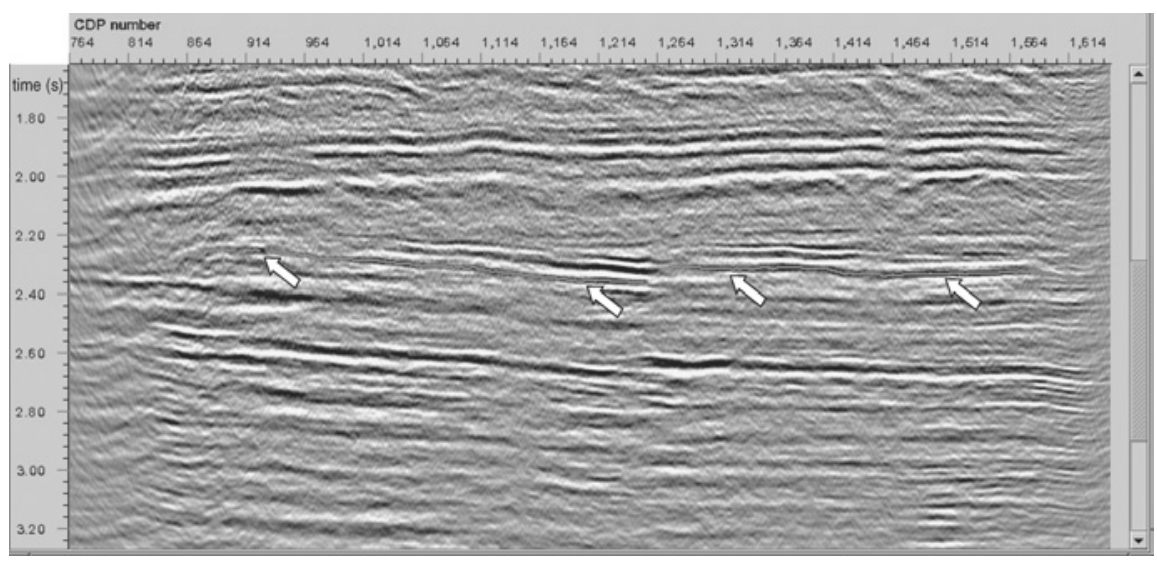

Figure 10. Time-migrated stack of North Sea data. For boundary-related internalmultiple removal, the multiple-generating reflector needs to be identified on a time section. For the North Sea data set under consideration, the multiple-generating boundary is picked by an automatic tracker (see the arrows).

(related to the DTS panels of Figure 11a) is shown in Figure 12. After one update, new CFP gathers and DTS panels are calculated. Figure $11 \mathrm{~b}$ shows that DTS panels now display flat events at zero differential time, indicating correct operators.

With the updated operators, a prestack volume of CFP gathers can be computed. Figure 13a shows a selection of these CFP gathers. The reflection event related to the selected boundary has its apex around $1.25 \mathrm{~s}$. The focusing operator times are also plotted in Figure 13a, and the traveltimes coincide with the considered reflection events. The events related to all boundaries above the selected boundary, and also the event from the boundary itself, need to be muted because they should not be involved in the prediction process. This muting can be done in a very straightforward manner along the focusing-operator times. Thus, the events visible in Figure 13b after muting correspond to the events from below the indicated level in Figure 10. The internal multiples are calculated by a spatial and temporal convolution of these muted CFP gathers for all points of the selected boundary.

In Figures 14 and 15 the complete process of surface-multiple removal and internal-multiple removal is demonstrated on this North Sea data set. Figure 14 displays the lower part of the stack before and after removal of prestack surface-related multiples. Good suppression has been achieved (as reported in Verschuur et al., 1992). However, in the target area (i.e., between 2.4 and 3.2 s) events are observed that do not coincide with the available well-log information. They are expected to be caused mainly by internal-multiple reflections.

The procedure of internal-multiple removal is applied in a boundary-related approach, using the CFP gathers after operator updating, as described above. The resulting stack of predicted multiples (but not matched in amplitude and phase) is shown in Figure 15a. The bright areas on the left and in the middle are recognizable in the data (Figure 14b). Adaptive subtraction in the shot-record domain and subsequent stacking yields the result in Figure 15b. A reduction of events can be observed on the left-hand side (between 3.0 and $3.5 \mathrm{~s}$ ) and on the right-hand side (around $3.0 \mathrm{~s}$ ). 


\section{FIELD-DATA EXAMPLE OF LAYER-RELATED APPROACH}

Finally, a demonstration of the layer-related approach to internal-multiple removal is shown on a North Sea field data set from the Voring area (courtesy Saga Petroleum A.S.). Since depth of the water bottom is approximately $1.3 \mathrm{~km}$, surface-related multiples arrive only below $3.5 \mathrm{~s}$, as visible in the stack display of Figure 16. In this stack, a set of strong reflectors can be observed around $2.5 \mathrm{~s}$. It is possible that this

a)

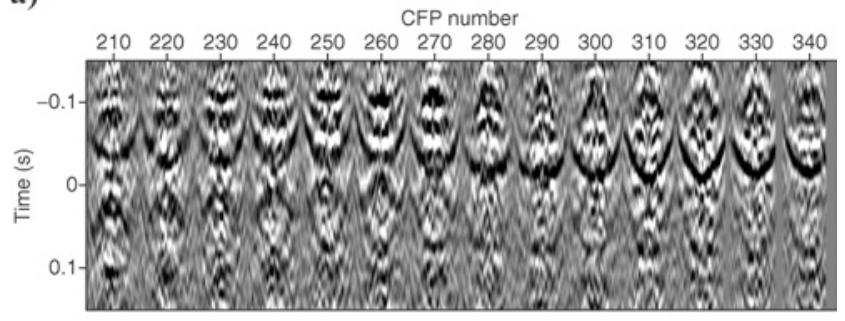

b)

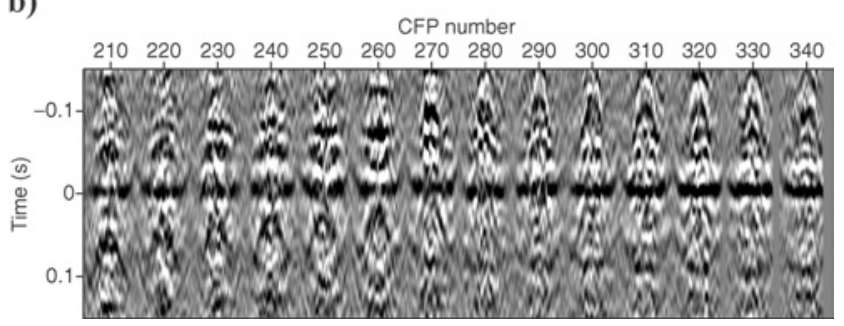

Figure 11. The process of operator updating involved in boundary-related internal-multiple prediction. (a) Initial operators based on the stacking velocities yield DTS panels with residual traveltime errors. (b) After one update, correct focusing operators are obtained; all DTS panels show aligned events at zero time.

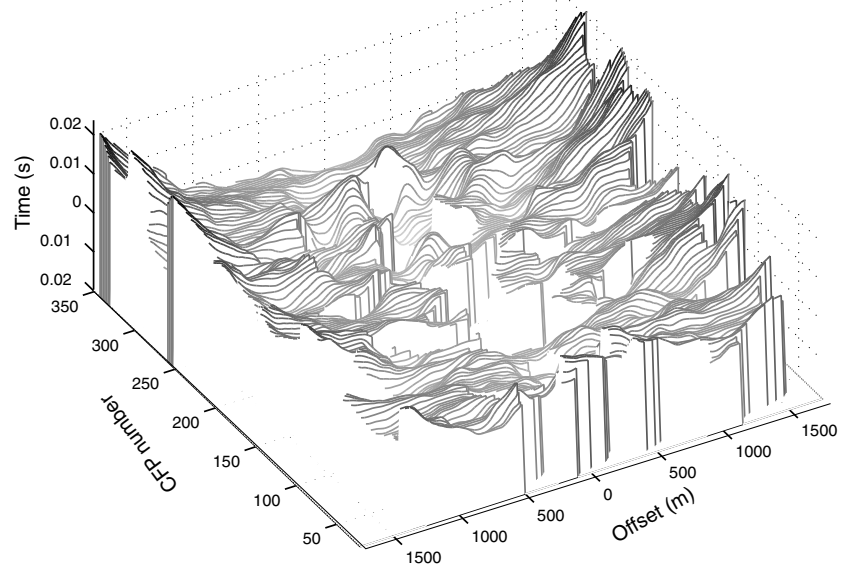

Figure 12. Using a multidimensional tracking algorithm, the traveltime errors can be found for all DTS panels. The tracked traveltime-error surface related to the DTS panels shown in Figure $11 \mathrm{a}$ is displayed in $3 \mathrm{D}$ view. Nontrackable parts are interpolated. The traveltime errors are used to update the focusing operators. a)

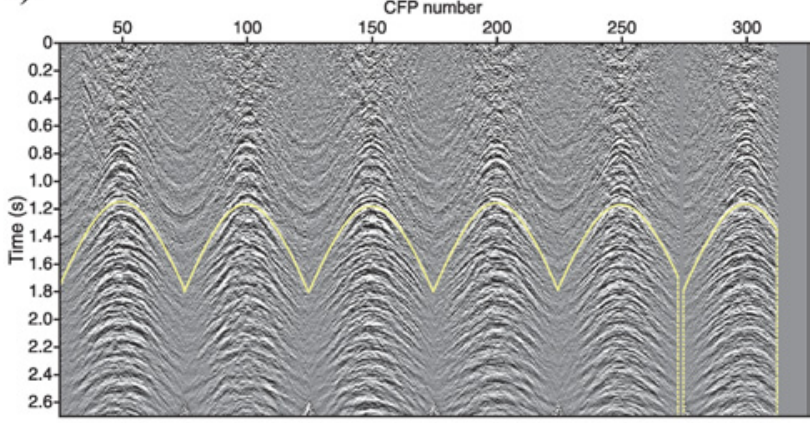

b)

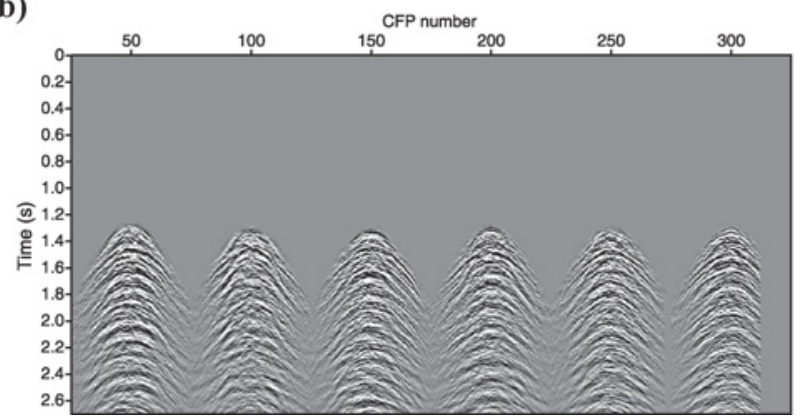

Figure 13. CFP gathers with focus points at the multiplegenerating boundary (a) before and (b) after muting along the focusing operator traveltimes. The muted CFP gathers are used to predict the boundary-related internal multiples, according to Figure $1 \mathrm{~b}$.

a)

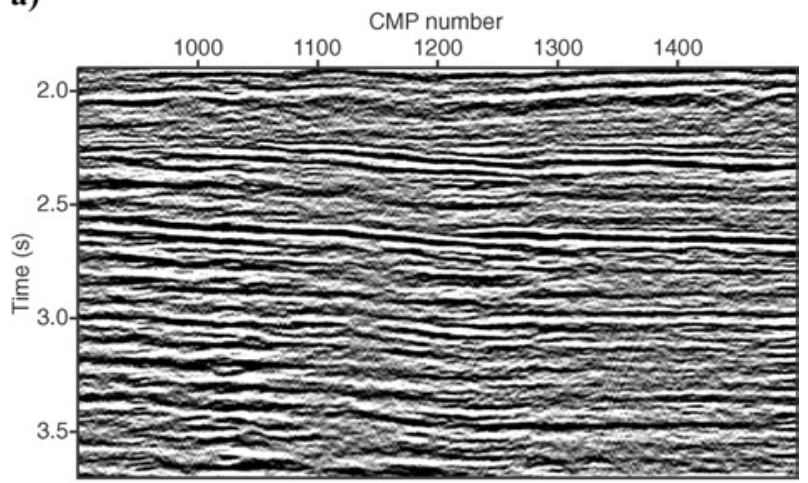

b)

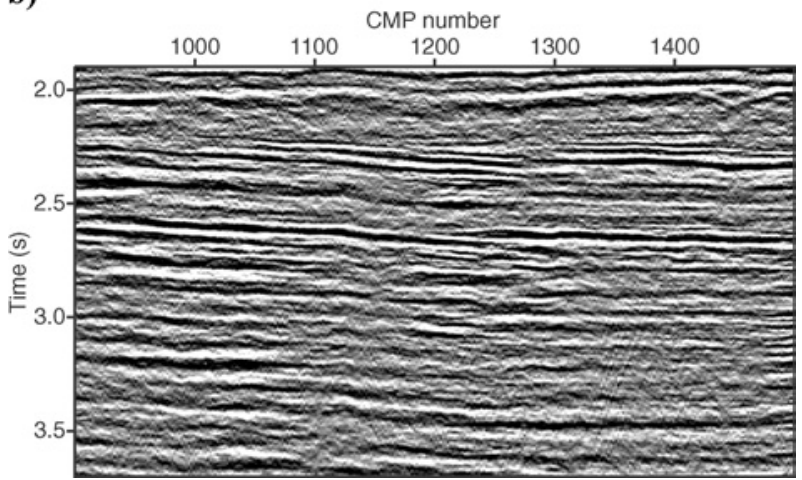

Figure 14. Result of surface-related-multiple removal on the North Sea data set. Note the enormous reduction of multiple energy between (a) the stack with all multiples and (b) the stack after multiple removal. 
structure will generate relatively strong internal multiples between these reflectors and the reflecting sediments above this level, including the sea bottom. This is confirmed between CMPs 600 and 900 around $3 \mathrm{~s}$ where a horizontal ringing is

a)

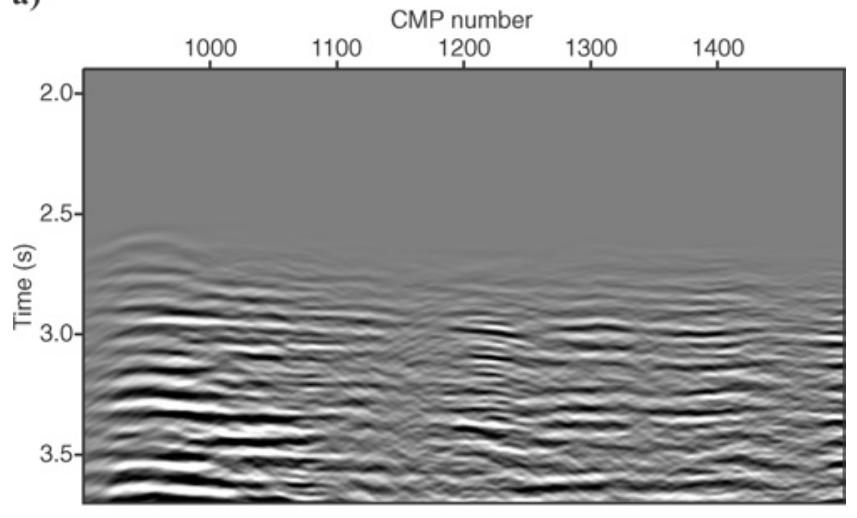

b)

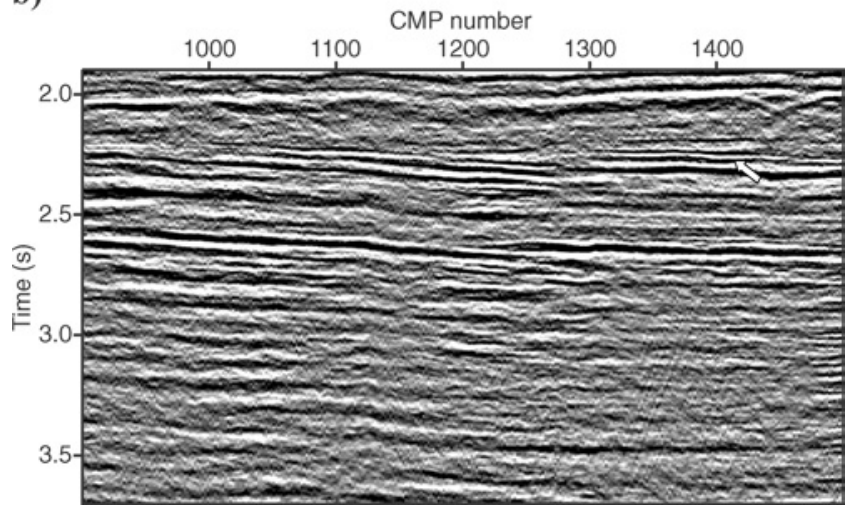

Figure 15. (a) Stack of unscaled predicted internal multiples and (b) the result of boundary-related internal-multiple removal on the North Sea data set. The arrow indicates the multiple-generating boundary (see also Figure 10). Compare the result of the multiple removal with the input data (Figure $14 \mathrm{~b})$ and note the reduction of events in the lower half.

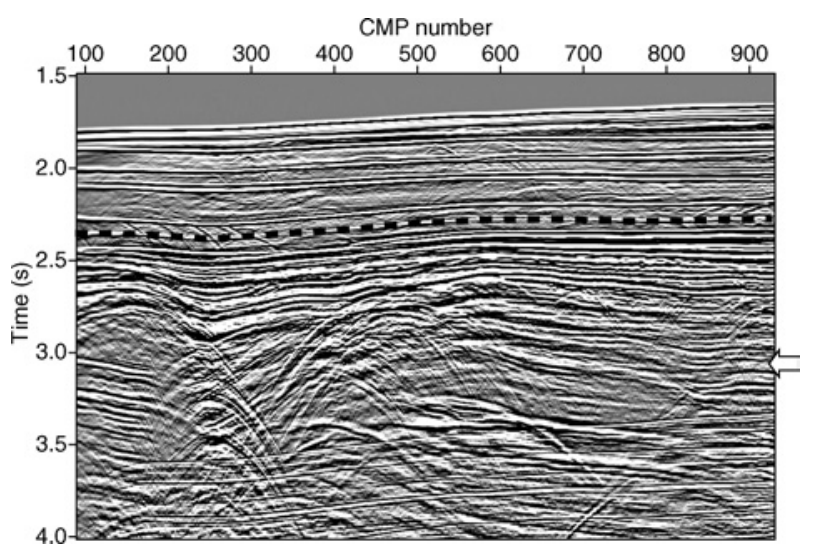

Figure 16. North Sea stack with all multiples. The dashed line indicates the level where internal multiples are calculated. The arrow indicates an area with primary internal-multiple interference below CMP 700. For this deep-water example, the surface multiples are present below $3.5 \mathrm{~s}$. observed, whereas primaries are dipping in that area. These internal multiples are of the first type (Figure 2b). Therefore, a suitable time level is chosen just above the structure that will serve as the lower boundary for predicting the layer-related internal multiples. This is shown with a dashed line in Figure 16. The same steps described in the physical-model data example on layer-related-multiple removal are carried out.

At the chosen time level, the focusing operators are constructed using the vertical time picks and the NMO velocities. No update of the operators is needed. From the CFP gathers, a muted version is obtained. Figure 17a shows a selection of these muted CFP gathers. Next, fully redatumed shot records (i.e., gridpoint gathers) need to be constructed at this level, from which the causal part is selected after time reversal. This produces the downward-scattering operators. Figure $17 \mathrm{~b}$ shows a set of these operators. Note that the deepest event in each gather corresponds to the water-bottom reflection. Finally, the actual prediction process is carried out by convolving the causal time-reversed gridpoint gathers twice with the muted CFP gathers (see Figure 1c).

In Figure 18, the result of adaptive subtraction of predicted multiples from three selected shot records is displayed. The predicted internal multiples match very well with events in

a)

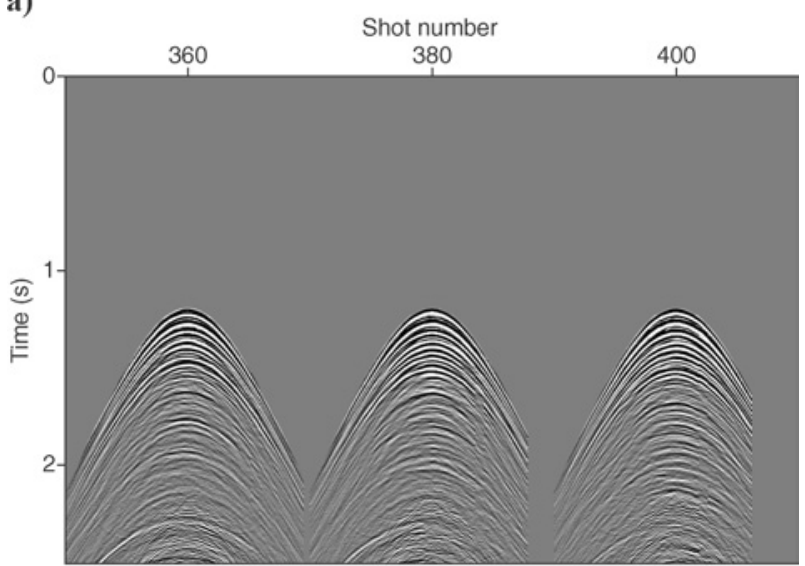

b)

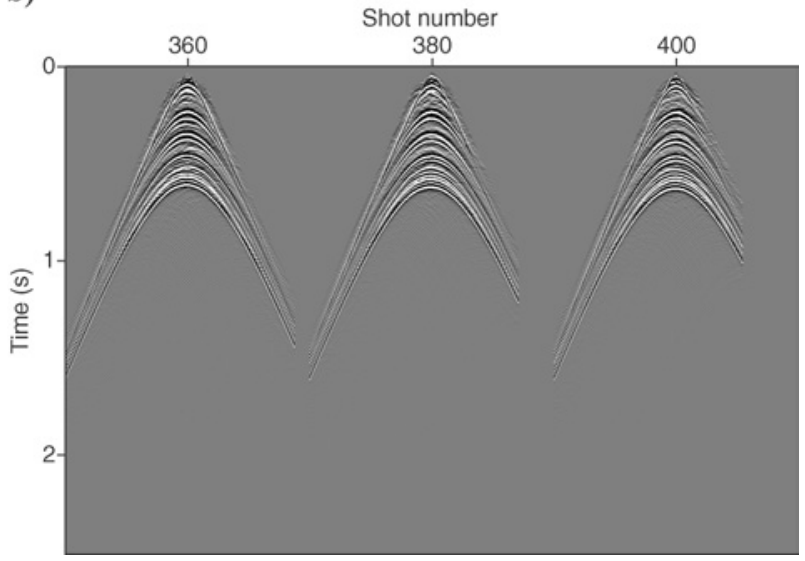

Figure 17. The prediction of layer-related internal multiples involves (a) the spatial and temporal convolution of muted CFP gathers with (b) the causal part of the time-reversed gridpoint gathers. 
the original shots (at the arrows). The effect of removing internal multiples can also be judged after stacking (Figure 19). After multiple attenuation, the interference patterns around 3 s between CMPs 600 and 900 are significantly reduced. The removed internal multiples can be observed in the difference plot in Figure 19c.

a)

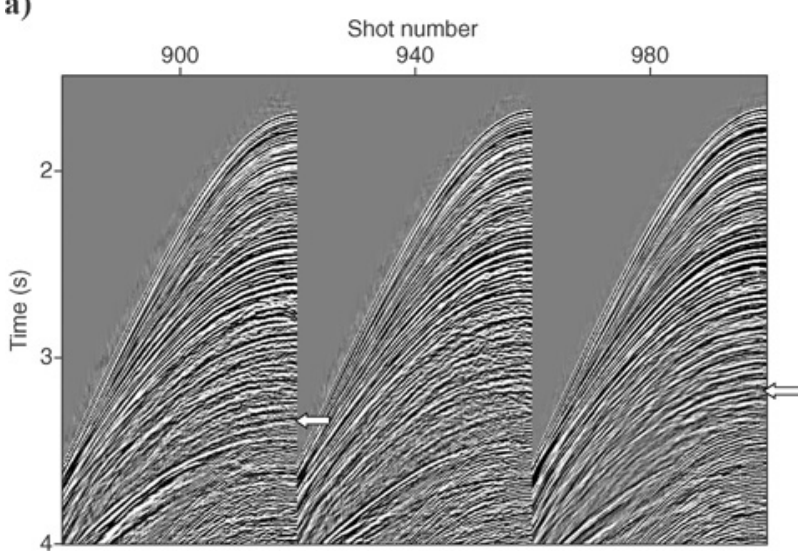

b)

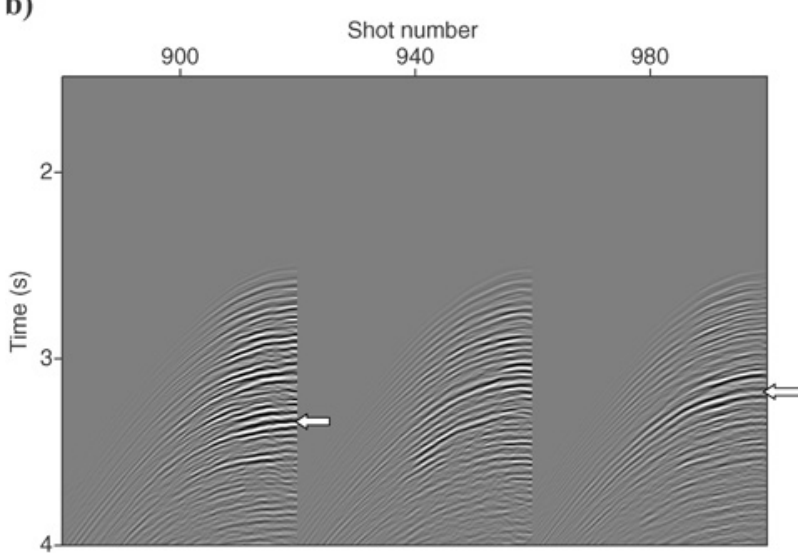

c)

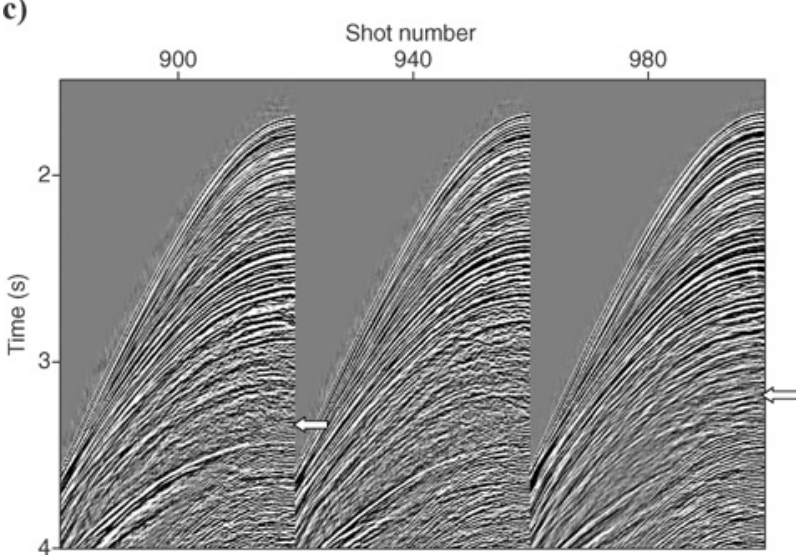

Figure 18. Demonstration of layer-related internal-multiple removal on three shot records from the Voring area field data set. (a) Shot records with internal multiples, (b) predicted internal multiples, and (c) shot records after multiple suppression. The arrows in (a) and (b) indicate a few internal multiple events that are clearly attenuated in (c).
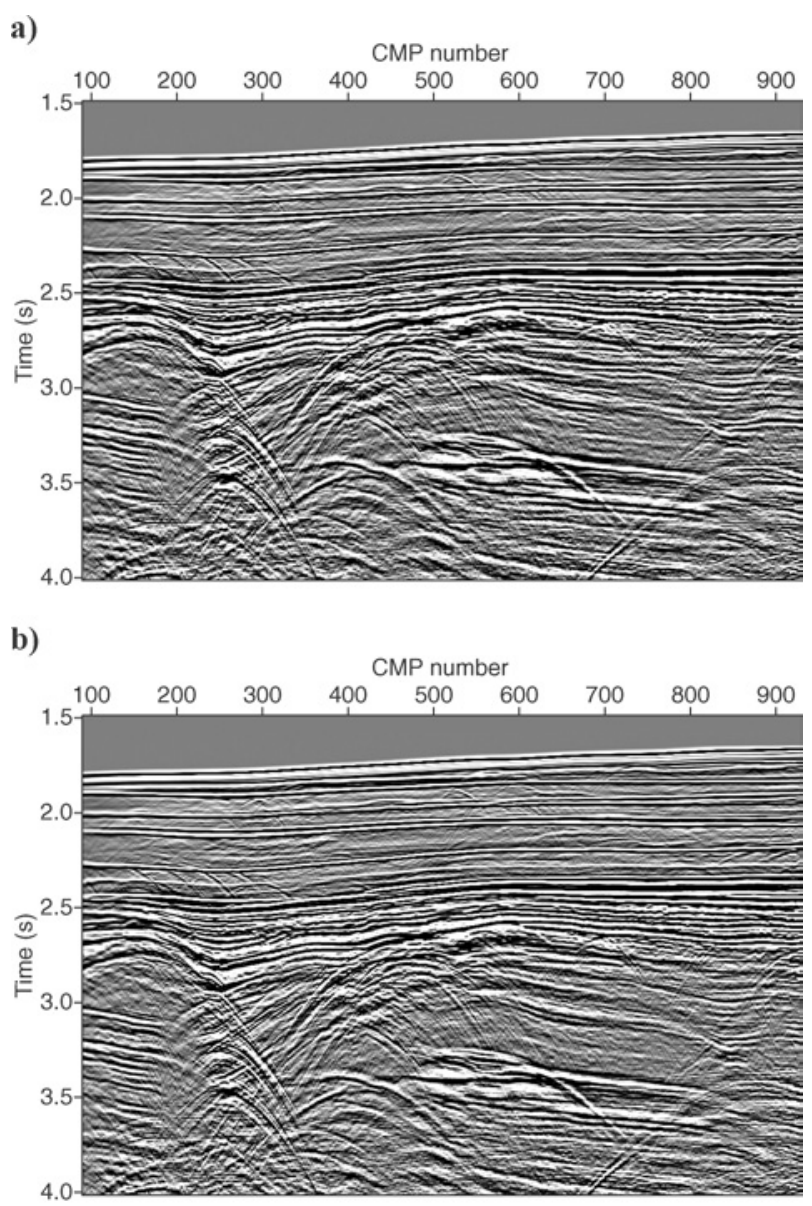

c)

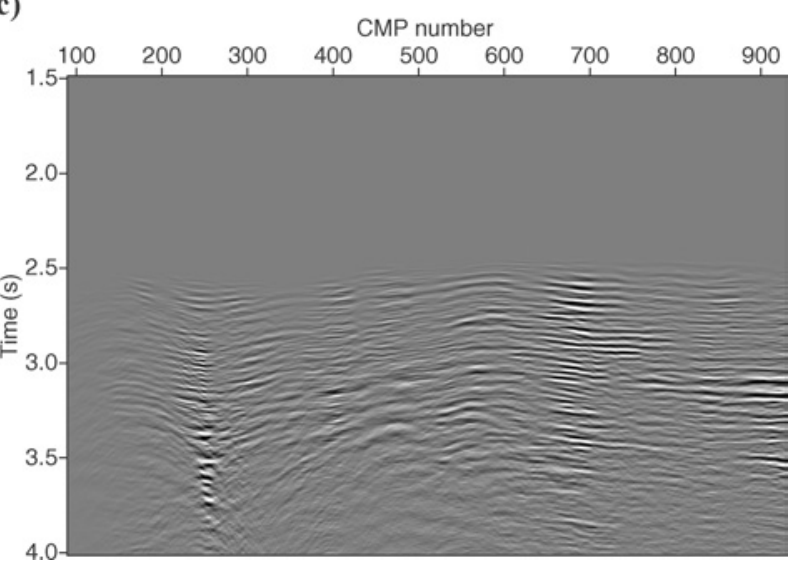

Figure 19. Result of layer-related internal-multiple removal at stack level. Stack after (a) surface multiple removed and (b) after layer-related internal-multiple removed. (c) Stack of internal multiples only - difference between (a) and (b). Note the improvement in the area below CMP 700 at $3.0 \mathrm{~s}$.

\section{DISCUSSION AND CONCLUSIONS}

Examples show that in the situation of one or more significant reflectors below the surface, internal multiples are observed with amplitudes comparable to primary reflections, which can confuse the interpretation. The proposed boundary-related as well as the layer-related approach to 
internal-multiple removal give good results on both physicalmodel and field data.

In the boundary-related approach, multiples related to one specific reflector are addressed. This approach requires the construction of CFP gathers, using focusing operators with correct traveltimes. Erroneous focusing operators can be updated with a data-driven procedure, thus avoiding the need for an exact velocity-depth model. The operator updating process involves picking traveltime error curves, which is facilitated with automatic tracking tools. The boundary-related approach should only be considered in situations of simple, easyto-track reflecting boundaries.

In the layer-related approach, multiples that pass the bottom of a complete layer at least four times (two times up, two times down) are addressed. The downward-scattering operator of this layer is determined in a data-driven way by computing the so-called gridpoint gather from the CFP gathers with sources and receivers at the lower boundary of the downwardscattering layer. In the layer-related approach, focusing operators are allowed to contain traveltime errors.

Both the boundary and the layer versions of internalmultiple removal require a muting process. In the CFP domain, the muting area is given directly by the focusing operator. Therefore, implementation of both algorithms is recommended in the CFP domain.

From a cost perspective, the boundary-related method involves more user interaction (updating of focusing operators) but in its final applications involves only one downward continuation step and one lateral convolution process. The layerrelated method requires only approximate focusing operators (no updating of focusing operators) but requires a double spatial convolution and double downward continuation of seismic data. Despite the extra calculation costs, the ease of use and the robustness of the layer-related approach make it preferable to the boundary-related approach in most situations.

This paper emphasizes the prediction of internal multiples. Once they are predicted, the subtraction of multiples is a process that needs a lot of attention as well. Because different subtraction procedures are available, the output of multiple suppression can vary, depending on the choice of the subtraction algorithm and its parameters.

Although the theory of internal-multiple removal is three dimensional in principle, our implementation was done only for a $2 \mathrm{D}$ acquisition geometry. A full 3D implementation requires a dense source and receiver sampling in both in-line and cross-line directions, which is not supported by current $3 \mathrm{D}$ data acquisition geometries. More research needs to be carried out to extend the current internal-multiple-prediction methodology to accommodate full $3 \mathrm{D}$ data.

\section{ACKNOWLEDGMENTS}

The authors thank Mohammed Hadidi, Helmut Jakubowicz, Panos Kelamis, Art Weglein, and Ken Matson for many fruitful and stimulating discussions. Also, the comments of the anonymous reviewers are highly appreciated. Furthermore, we acknowledge Saga Petroleum A.S. (currently part of Norsk Hydro) for use of the North Sea field data sets, and the Netherlands Geoscience Institute TNO for its support in acquiring the physical-model data.

\section{REFERENCES}

Berkhout, A. J., 1982, Seismic migration, imaging of acoustic energy by wave field extrapolation, A: Theoretical aspects, 2 nd ed.: Elsevier Science Publishing Co.

. 1997a, Pushing the limits of seismic imaging — Part I: Prestack migration in terms of double dynamic focusing: Geophysics, 62, 937-953.

— $1997 \mathrm{~b}$, Pushing the limits of seismic imaging — Part II: Integration of prestack migration, velocity estimation and AVO analysis: Geophysics, 62, 954-969.

, 1999, Multiple removal based on the feedback model: The Leading Edge, 18, 127-131.

Berkhout, A. J., and D. J. Verschuur, 1997, Estimation of multiple scattering by iterative inversion - Part I: Theoretical considerations: Geophysics, 62, 1586-1595.

,+ 2005 , Removal of internal multiples with the common-focuspoint (CFP) approach - Part 1: Explanation of the theory: Geophysics, 70, V45-V60.

Blacquière, G., A. W. F. Volker, and L. Ongkiehong, 1999, 3-D physical modeling for acquisition geometry studies: 69th Annual International Meeting, SEG, Expanded Abstracts, 12121215.

Bolte, J. F. B., and D. J. Verschuur, 1998, Aspects of focusing operator updating: 68th Annual International Meeting, SEG, Expanded Abstracts, 1604-1607.

Bolte, J. F. B., R. J. Prein, D. J. Verschuur, J. W. Thorbecke, and P. L. A. Winthaegen, 2000, Volume visualization and automatic tracking in CFP-related processing: 70th Annual International Meeting, SEG, Expanded Abstracts, 529-532.

Hadidi, M. T., and D. J. Verschuur, 1997, Removal of internal multiples: Field data examples: 59th Annual International Meeting, European Association of Geoscientists and Engineers, Extended Abstracts, A013.

Jakubowicz, H., 1998, Wave equation prediction and removal of interbed multiples: 60th Annual International Meeting, European Association of Geoscientists and Engineers, Extended Abstracts, Session 1-28.

Kelamis, P. G., K. E. Erickson, D. J. Verschuur, and A. J. Berkhout, 2002, Velocity-independent redatuming: A new approach to the near-surface problem in land seismic data processing: The Leading Edge, 21, 730-735.

Kennett, B. L. N., 1979, The suppression of surface multiples on seismic records: Geophysical Prospecting, 27, 584-600.

Koek, E. A., G. Faber, and A. J. Berkhout, 1995, 3-D data acquisition research with the Delft physical modeling facility: 65th Annual International Meeting, SEG, Expanded Abstracts, 747748.

Matson, K. H., D. Corrigan, A. B. Weglein, and C. Y. Young, 1999, Inverse scattering internal multiple attenuation: Results from complex synthetic and field data examples: 69th Annual International Meeting, SEG, Expanded Abstracts, 1060-1063.

Morton, S. A., 1996, Automating prestack migration analysis using common-focal-point gathers: 66th Annual International Meeting, SEG, Expanded Abstracts, 411-414.

Riley, D. C., and J. F. Claerbout, 1976, 2-D multiple reflections: Geophysics, 41, 592-620.

Spagnolini, U., and V. Rampa, 1999, Multitarget detection/tracking for monostatic ground penetrating radar: Application to pavement profiling: IEEE Transactions on Geoscience and Remote Sensing, 37, no. 1, 383-394.

Thorbecke, J. W., 1997, Common focus point technology: Ph.D. dissertation, Delft University of Technology.

Verschuur, D. J., 1991, Surface-related multiple elimination: An inversion approach: Ph.D. dissertation, Delft University of Technology.

Verschuur, D. J., and A. J. Berkhout, 1997, Estimation of multiple scattering by iterative inversion - Part II: Practical aspects and examples: Geophysics, 62, 1596-1611.

Verschuur, D. J., A. J. Berkhout, and C. P. A. Wapenaar, 1992, Adaptive surface-related multiple elimination: Geophysics, 57, 11661177.

Weglein, A. B., F. A. Gasparotto, P. M. Carvalho, and R. H. Stolt, 1997, An inverse scattering series method for attenuating multiples in seismic reflection data: Geophysics, 62, 1975-1989. 